\title{
Niti CAR 27 Versus a Conventional End-to-End Anastomosis Stapler in a Laparoscopic Anterior Resection for Sigmoid Colon Cancer
}

\author{
Seung-Jin Kwag, Jun-Gi Kim ${ }^{1}$, Won-Kyung Kang ${ }^{1}$, Jin-Kwon Lee ${ }^{1}$, Seong-Taek Oh ${ }^{1}$ \\ Department of Surgery, Gyeongsang National University, Postgraduate School of Medicine, Jinju; ${ }^{1}$ Department of Surgery, The Catholic \\ University of Korea, Seoul St. Mary’s Hospital, Seoul, Korea
}

Purpose: The Niti CAR 27 (ColonRing) uses compression to create an anastomosis. This study aimed to investigate the safety and the effectiveness of the anastomosis created with the Niti CAR 27 in a laparoscopic anterior resection for sigmoid colon cancer.

Methods: In a single-center study, 157 consecutive patients who received an operation between March 2010 and December 2011 were retrospectively assessed. The Niti CAR 27 (CAR group, 63 patients) colorectal anastomoses were compared with the conventional double-stapled (CDS group, 94 patients) colorectal anastomoses. Intraoperative, immediate postoperative and 6-month follow-up data were recorded.

Results: There were no statistically significant differences between the two groups in terms of age, gender, tumor location and other clinical characteristics. One patient (1.6\%) in the CAR group and 2 patients $(2.1 \%)$ in the CDS group experienced complications of anastomotic leakage $(\mathrm{P}=0.647)$. These three patients underwent a diverting loop ileostomy. There were 2 cases $(2.1 \%)$ of bleeding at the anastomosis site in the CDS group. All patients underwent a follow-up colonoscopy (median, 6 months). One patient in the CAR group experienced anastomotic stricture ( $1.6 \%$ vs. $0 \%$; $\mathrm{P}=0.401)$. This complication was solved by using balloon dilatation.

Conclusion: Anastomosis using the Niti CAR 27 device in a laparoscopic anterior resection for sigmoid colon cancer is safe and feasible. Its use is equivalent to that of the conventional double-stapler.

Keywords: Anastomotic leak; Colorectal; Compression; Niti

\section{INTRODUCTION}

Complications such as leakage, bleeding, and stricture can develop following colorectal anastomosis. In particular, anastomotic leakage is a major problem that contributes not only to postoperative morbidity and mortality but also to local recurrence and a poor

Received: April 29, 2013 - Accepted: July 24, 2013

Correspondence to: Seong-Taek Oh, M.D.

Department of Surgery, The Catholic University of Korea, Seoul St. Mary's

Hospital, 222 Banpo-daero, Seocho-gu, Seoul 137-701, Korea

Tel: +82-2-2258-6100, Fax: +82-2-595-2822

E-mail: stoh@catholic.ac.kr

(C) 2014 The Korean Society of Coloproctology

This is an open-access article distributed under the terms of the Creative Commons Attribution NonCommercial License (http://creativecommons.org/licenses/by-nc/3.0) which permits unrestricted non-

commercial use, distribution, and reproduction in any medium, provided the original work is properly cited. prognosis [1,2]. Many comparison studies of laparoscopic and open surgery for rectal cancer have shown no significant differences in terms of anastomotic leakage [3, 4]. The stapling techniques used in colorectal anastomosis in anterior resection have gained widespread acceptance over hand-sewn anastomosis [5]. However, the stapled colorectal anastomosis has a higher rate of stenosis compared with the hand-sewn anastomosis [6], and a meta-analysis showed that the stapled anastomosis had a higher rate of stricture compared with hand-sewn anastomosis for colorectal anastomoses $(\mathrm{P}<0.028)$ [7].

Recently, a compression anastomosis ring, Niti CAR 27 (NiTi Surgical Solutions, Netanya, Israel), was introduced. Some studies reported the safety and efficacy of this novel instrument [8-10]. The Niti CAR 27 consists of a ring with Nitinol leaf springs and an applier similar to that used with standard surgical circular staples. Nitinol is an advanced metal alloy that exhibits "shape mem- 
ory," which is the ability to return to its original shape after deformation. After the ring is set during surgery, it returns to its original closed position, accommodating variations in tissue thickness. The natural healing process generates healthy tissue along the outer perimeter of the ring. The necrotic tissue and the ring are expected to detach within approximately two weeks, and safely pass through the digestive tract. The aim of the current study was to evaluate the safety and effectiveness of the Niti CAR 27 device compare to the conventional stapler in laparoscopic surgery for sigmoid colon cancer.

\section{METHODS}

In this retrospective study, we included only patients with sigmoid colon cancer who underwent a laparoscopic anterior resection. This study was approved by the Institutional Review Board of Seoul St Mary's Hospital on September 05, 2011 (KC11RGSI0676). Curative operations were performed by one experienced colorectal surgeon (S.T.O.) who specialized in colorectal surgery at Seoul St. Mary's Hospital. All of the data were retrieved from our electronic medical record system, and a retrospective analysis was performed.

Between March 2010 and February 2012, 63 patients (44 males) underwent a laparoscopic anterior resection and compression anastomosis with the Niti CAR 27 device (CAR group). These CAR group patients were retrospectively compared with patients who underwent a laparoscopic conventional double-stapled anastomosis (CDS group: 48 procedures performed with a Curved Intraluminal Stapler 29 [Ethicon, Edinburgh, UK] and 46 with a DST EEA 31 [Covidien, Norwalk, CT, USA]) between March 2010 and February 2012. Inclusion criteria of this study were a diagnosis of sigmoid colon cancer, no combination operation, and no preoperative chemoradiotherapy. In both groups, all of the anastomoses were created above the anterior peritoneal reflection. The presence of underlying disease (hypertension, diabetes mellitus, coronary artery disease, or cerebro-vascular disease) was investigated.

The following short-term results were collected retrospectively: the patients' demographic information, operative details, postoperative morbidity and mortality, and the pathological outcomes derived from the patients' medical records. Anastomotic leakage was defined as the presence of clinical symptoms of sepsis, including abdominal pain, tenderness, rebound tenderness or fever. Clinical anastomotic leakage was diagnosed by the presence of gas or fecal discharge from the pelvic drain or fluid/air bubbles surrounding the anastomosis as viewed on computed tomography scans. After a median of six months postoperatively, we performed a routine follow-up colonoscopy. Anastomotic stricture was defined as the inability to insert a standard 12-mm diameter colonoscope, which required balloon dilatation.

In both groups, bowel preparation with a polyethylene-glycol electrolyte lavage solution 24 hours before surgery and administration of antibiotic prophylaxis with 250-mg oral metronidazole twice were included in the standard preoperative protocols. All pa- tients received $1 \mathrm{~g}$ of cefminox intravenously at the beginning of surgery and postoperatively twice daily for 1 day.

Laparoscopic mobilization of the left colon was performed using a medial-to-lateral approach, and the inferior mesenteric artery was ligated at a high level. The splenic flexure was not routinely taken down. If there was tension in the anastomosis, we performed mobilization of the splenic flexure. An intracorporeal transection of the bowel distal to the tumor was performed using an endoscopic linear stapler. The specimen was extracted through the left lower quadrant port, which was extended to approximately $4 \mathrm{~cm}$ in length. The Niti CAR 27 device was manipulated according to the manufacturer's instructions. After the anvil of the Niti CAR 27 had been secured, the bowel was returned to the peritoneal cavity, and the pneumoperitoneum was re-established. After the mounted ring with its loader had been immersed in cold saline for several minutes, the ring was loaded onto the distal end of the main shaft and inserted through the anus, making an intracorporeal end-toend anastomosis. Because this device is temperature dependent, warm saline was instilled around the anastomosis after the device had been fired for a quicker and more secure return to its original shape. For the CDS procedure, we used either the Curved Intraluminal Stapler 29 or the DST EEA 31 device. Other procedures were the same as the CAR procedure. Finally, we tested for air and fluid leakage through the anus in both groups. If the leakage test was positive, we performed a diverting ileostomy. Seven patients (2 in the CAR group and 5 in the CDS group) who received a diverting ileostomy during the operation were excluded from this study.

The statistical analysis was performed using PASW ver. 18.0 (SPSS Inc., Chicago, IL, USA). The chi-square and the t-tests were used to compare the data. A P-value of less than 0.05 was considered statistically significant.

\section{RESULTS}

A total of 157 patients (CAR group, 63; CDS group, 94) received a laparoscopic anterior resection for sigmoid colon cancer. The patient demographic and clinical characteristics are shown in Table 1. There were no statistically significant differences between the two groups in terms of age, gender, body mass index and distance from the anal verge. The most common comorbidities included hypertension (39.5\% of patients), diabetes mellitus (16.6\%) and coronary artery disease (3.8\%). The American Statistical Association score was 1 or 2 in both groups.

The surgical and oncologic outcomes are shown in Table 2. There was no difference in the mean operative times between the two groups (163 \pm 39 minutes for the CAR group and $167 \pm 34$ minutes for the CDS group, $\mathrm{P}=0.550)$. Full mobilization of the splenic flexure was performed in 12 patients $(19.0 \%)$ in the CAR group and 25 patients $(26.6 \%)$ in the CDS group. In the CAR group, $51(81.0 \%)$ and 12 patients (19.0\%) underwent transection at the distal rectum by using one and two linear staples, respectively. In the CDS group, 74 (78.7\%) and 20 patients (21.3\%) un- 
Table 1. Patient demographics and clinical characteristics

\begin{tabular}{llll}
\hline Characteristic & $\begin{array}{c}\text { Niti CAR 27 } \\
(\mathrm{n}=63)\end{array}$ & $\begin{array}{c}\text { Conventional } \\
(\mathrm{n}=94)\end{array}$ & P-value \\
\hline Age $(\mathrm{yr})$ & 60.6 & 59.7 & 0.765 \\
Gender & & & \\
$\quad$ Male/female & $44 / 19$ & $57 / 37$ & 0.308 \\
Body mass index $\left(\mathrm{kg} / \mathrm{m}^{2}\right)$ & 23.8 & 24.5 & 0.128 \\
Distant from anal verge $(\mathrm{cm})$ & 20.4 & 21.4 & 0.138 \\
Underlying disease & $37(58.7)$ & $41(50.0)$ & 0.329 \\
$\quad$ Hypertension & $21(33.3)$ & $41(43.6)$ & 0.196 \\
$\quad$ Diabetes mellitus & $10(15.9)$ & $17(18.1)$ & 0.719 \\
Coronary artery disease & $5(7.9)$ & $1(1.1)$ & 0.028 \\
Others & $3(4.8)$ & $2(2.1)$ & 0.357 \\
ASA score & & & 0.116 \\
1 & $25(39.7)$ & $31(33.0)$ & \\
\hline 2 & $33(52.4)$ & $61(64.9)$ & \\
3 & $5(7.9)$ & $2(2.1)$ & \\
\hline
\end{tabular}

Values are presented as number (\%).

ASA, American Statistical Association.

Table 3. Anastomotic leakage and bleeding

\begin{tabular}{lccc}
\hline & $\begin{array}{c}\text { Niti CAR 27 } \\
(\mathrm{n}=63)\end{array}$ & $\begin{array}{c}\text { Conventional } \\
(\mathrm{n}=94)\end{array}$ & P-value \\
\hline Anastomotic leakage & $1(1.6)$ & $2(2.1)$ & 0.647 \\
Bleeding on anastomosis site & $0(0)$ & $2(2.1)$ & 0.516 \\
\hline
\end{tabular}

Values are presented as number (\%).

derwent transections by using one and two linear staples, respectively $(\mathrm{P}=0.841)$. The times of first passage of flatus, and first soft diet and the length of postoperative hospital stay were similar for both groups.

For both groups, no surgical mortality or readmission occurred within the first 30 postoperative days. Table 3 shows the result of anastomotic complications such as leakage and bleeding. Anastomotic leakage occurred in 1 patients $(1.6 \%)$ in the CAR group 2 days postoperatively and 2 patients $(2.1 \%)$ in the CDS group $(\mathrm{P}=$ $0.647) 3$ and 7 days postoperatively, respectively. These three patients underwent a diverting ileostomy by using a laparoscopic method. The patient with an anastomotic leakage in the CAR group was a 35-year-old male (stage T3N2M0) with distal sigmoid colon cancer. He had no underlying disease. There were no significant risk factors for anastomotic leakage, such as bleeding during the operation. However, his rectum was transected by using two linear staple. He underwent laparoscopic reoperation, and a primary suture closure of the anastomotic defect (Fig. 1) with a temporary diverting loop ileostomy was performed. He was aware that the colon ring was expelled two months after his reoperation. He underwent a follow-up sigmoidoscopy three months postoperatively. The anastomosis site was well-healed, and there was no
Table 2. Surgical and oncologic data

\begin{tabular}{llll}
\hline Variable & $\begin{array}{c}\text { Niti CAR 27 } \\
(\mathrm{n}=63)\end{array}$ & $\begin{array}{c}\text { Conventional } \\
(\mathrm{n}=94)\end{array}$ & P-value \\
\hline Operative time (min) & $163 \pm 39$ & $167 \pm 34$ & 0.550 \\
No. of linear staples & & & 0.841 \\
1 & $51(81.0)$ & $74(78.7)$ & \\
\hline 2 & $12(19.0)$ & $20(21.3)$ & \\
Mobilization of splenic flexure & $12(19.0)$ & $25(26.6)$ & 0.339 \\
Gas out (day) & 1.7 & 1.8 & 0.478 \\
Diet (day) & 2.5 & 2.8 & 0.150 \\
Length of hospital stay (day) & 5.9 & 6.2 & 0.104 \\
T stage & & & 0.214 \\
$\quad$ T1,T2 & $21(33.3)$ & $48(51.1)$ & \\
$\quad$ T3,T4 & $42(66.7)$ & $46(48.9)$ & \\
Tumor size (cm) & 3.9 & 3.6 & 0.275 \\
Distal free margin (cm) & 5.2 & 5.6 & 0.195 \\
\hline
\end{tabular}

Values are presented as mean \pm standard deviation or number (\%).

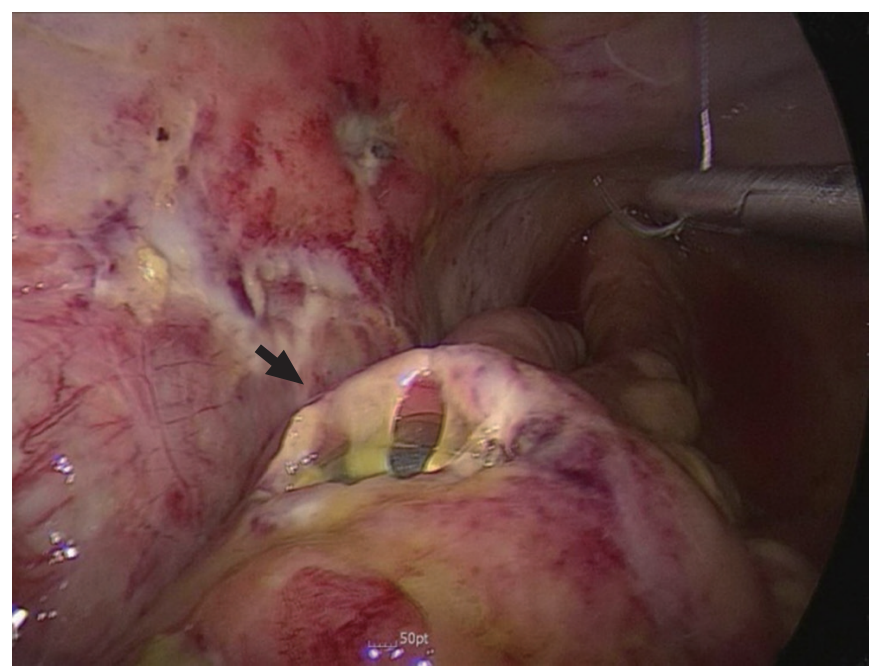

Fig. 1. Intraoperative image from the laparoscope showing that the colon ring was exposed (black arrow; dehiscence of anastomosis).

stricture. He underwent a repair of the loop ileostomy four months postoperatively. There were no bleeding complications in the CAR group. However, in the CDS group, two patients (2.1\%) with hypertension and coronary heart disease experienced bleeding at the anastomosis site. These patients complained of hematochezia on the day of the operation or on the first postoperative day. Sigmoidoscopy was performed immediately, and bleeding on the anastomosis site was observed. The bleeding was successfully treated by endoscopic clipping.

After a median of six months postoperatively, all patients of both groups underwent a follow-up colonoscopy. We observed wide and patent anastomoses without metallic foreign material in the 

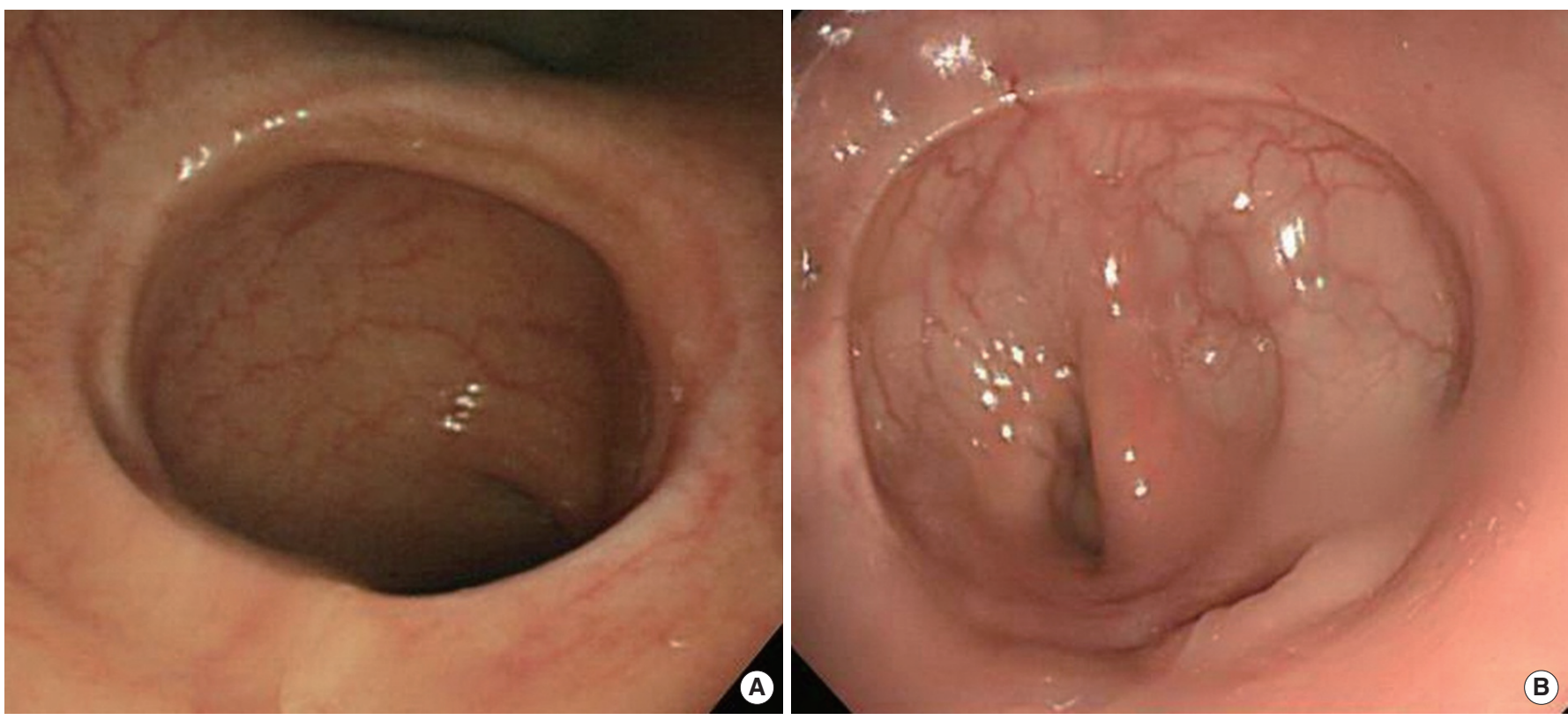

Fig. 2. Colonoscopic views at the 6 month follow-up. (A) Wide and circumferential anastomosis without any retained foreign material constructed by Niti CAR 27. (B) Non-ova-shaped stapled anastomoses with metallic clips

Table 4. Results of colonoscopy at a median 6-month follow-up

\begin{tabular}{lccc}
\hline & $\begin{array}{c}\text { Niti CAR 27 } \\
(\mathrm{n}=63)\end{array}$ & $\begin{array}{c}\text { Conventional } \\
(\mathrm{n}=94)\end{array}$ & P-value \\
\hline $\begin{array}{c}\text { Height of anastomosis } \\
\text { (from anal verge) }(\mathrm{cm})\end{array}$ & 12.6 & 13.1 & 0.471 \\
\begin{tabular}{l} 
Anastomotic stricture, $\mathrm{n}(\%)$ \\
\hline
\end{tabular} & $1(1.6)$ & $0(0)$ & 0.401 \\
\hline
\end{tabular}

CAR group, which contrasted with non-ova- shaped stapled anastomoses with metallic clips in the CDS group (Fig. 2). In the CAR group, one patients had an anastomotic stricture (1.6\% vs. $0 \%$; $\mathrm{P}=$ 0.401); however, he did not complain of any specific symptom. This complication was solved by using balloon dilatation. The dilatation was conducted up to $18 \mathrm{~mm}$ (Table 4 ).

\section{DISCUSSION}

The concept of tissue compression during colorectal anastomosis is not new, and previous attempts at tissue compression have used devices such as the Murphy button, the biofragmentable anastomotic ring, and AKA-2 devices [11-13]. However, these devices have not gained broad acceptance among colorectal surgeons because foreign material is retained within the tissue, the lumen is narrowed, and necrosis occurs at the anastomotic site [11, 14]. Thus, the use of these devices has become extremely limited. Recently, a novel compression anastomosis ring, the Niti CAR 27 device, was introduced, and the safety of this device has been demonstrated in animal studies [15-17]. The CAR 27 device was approved by the U.S. Food and Drug Administration for intestinal anastomosis in August 2006 and by the Korea Food and Drug
Administration in May 2009. Nitinol (nickel titanium, Naval Ordnance Laboratory), a metal alloy of nickel and titanium, has two advantageous physical properties: shape memory and super-elasticity $[8,18,19]$. The CAR 27 device itself and its handling are very similar to the currently-used circular staplers and their handling. However, there are some essential differences that may lead to problems with and malfunctions of the device [19]. For example, in the case of the CAR 27 device, reopening of the device after closure is impossible; moreover, pulling the trigger after firing is very dangerous. However, no malfunctions of the device occurred during this study. Also, no patients complained of pain or discomfort while the ring was in place. Retention of the colon ring could be a serious problem. Because fecal impaction may occur at the proximal of the ring and stricture may occur due to leakage, removing the ring is difficult. Thus, the ring must be removed before stoma closure if the patient had a diverting stoma because of leakage. Tulchinsky et al. [9] recommended digital removal of the ring when it was not expelled spontaneously in a diverted patient. Dauser et al. [20] removed the ring by using an endoscopic method. However, Koo et al. [8] emphasized that the artificial method seemed to be an unnecessary maneuver. In the case of their patient who received a diverting ileostomy, the ring was not expelled, but was retained, during the diversion; it was removed spontaneously after the ileostomy closure. In this study, the patient who experienced anastomotic leakage was aware that the ring was expelled two months after the operation.

Previously, there have been reports about clinical experiences with the use of the CAR 27 device in colorectal anastomosis. A prospective multicenter study comparing 10 anastomoses using 
CAR 27 with 13 stapled anastomoses reported that the safety and efficacy of the CAR 27 in humans were comparable to those of the standard stapling technology and that no anastomotic leakages had occurred [9]. However, anastomotic strictures were not mentioned, and the sample size was too small. A nonrandomized prospective study of the CAR 27 device involving 23 patients who underwent a left-sided colectomy reported anastomotic leakage in one patient $(4.3 \%)$ and stricture in two patients (8.6\%) [10]. Another nonrandomized prospective study showed that among 62 patients who underwent rectal anastomosis, there were no anastomotic leakages and two anastomotic strictures (3.3\%) [20]. A recent study in 66 patients who underwent a laparoscopic colectomy for left-sided colon cancer or an adenoma reported anastomotic leakage in one patient (1.5\%) [8].

The current study was designed to evaluate the safety and efficacy of the CAR 27 device in terms of anastomotic complications: leakage, bleeding and stenosis. With regard to anastomotic leakage, our results demonstrated that the CAR 27 device was not worse than the conventional device. In this study, there was one patient $(1.6 \%)$ with anastomotic leakage in the CAR group. This result is within the range expected based on other reported rates of anastomotic leakage (from $2.9 \%$ to $15.3 \%$ ) after a laparoscopic colorectal anastomosis $[10,21,22]$. Although no uniform definition of anastomotic stenosis exists [6], we defined it as an inability to traverse the anastomosis with a colonoscope with a $12-\mathrm{mm}$ diameter. In this study, there was one patient (1.6\%) with anastomotic stricture. This result was lower than that in other reports (from $3.3 \%$ to $8.7 \%$ ) $[10,20]$. The patient with anastomotic stricture had no problem to defecate. Based on the granulation tissue of anastomosis site observed during colonoscopy, we assume that the stricture of anastomosis was due to an undetected anastomotic leakage after surgery. A recent study showed that a symptomatic anastomotic stenosis, necessitating an endoscopic dilatation, was found in 12 patients (17.6\%) after using a conventional stapler in a laparoscopic sigmoidectomy for diverticular disease [23].

There was no bleeding at the anastomosis site in the CAR group. Certainly, bleeding at the anastomosis site does not seem to be an issue when using a compression anastomotic device [12, 13, 20], an observation that is supported by the results of the present study. We can consider using the Niti CAR 27 device in patients who have a high risk of bleeding. Middle and lower rectal tumors are risk factors for anastomotic leakage in laparoscopic colorectal surgery $[21,22,24]$. Thus, we only used the CAR 27 device in cases of sigmoid colon cancer. However, this restriction is also a limitation of this study. Therefore, we need to investigate and better understand the indications for use of the CAR 27 in patients with rectal cancer. Another limitation of this study is its retrospective, nonrandomized design. However, to reduce bias, we only enrolled patients who had the same diseases and who underwent the same surgical procedures in this study.

In conclusion, we demonstrated that anastomosis with the Niti CAR 27 device in a laparoscopic anterior resection for sigmoid colon cancer is safe and effective. There were no differences in the anastomotic complication rates of leakage and stricture between the CAR and the CDS groups. Large and randomized prospective studies are necessary to validate these results on the use of the Niti CAR 27.

\section{CONFLICT OF INTEREST}

No potential conflict of interest relevant to this article was reported.

\section{REFERENCES}

1. Bell SW, Walker KG, Rickard MJ, Sinclair G, Dent OF, Chapuis $\mathrm{PH}$, et al. Anastomotic leakage after curative anterior resection results in a higher prevalence of local recurrence. Br J Surg 2003;90: 1261-6.

2. Jung SH, Yu CS, Choi PW, Kim DD, Park IJ, Kim HC, et al. Risk factors and oncologic impact of anastomotic leakage after rectal cancer surgery. Dis Colon Rectum 2008;51:902-8.

3. Feliciotti F, Guerrieri M, Paganini AM, De Sanctis A, Campagnacci $R$, Perretta $S$, et al. Long-term results of laparoscopic versus open resections for rectal cancer for 124 unselected patients. Surg Endosc 2003;17:1530-5.

4. Anthuber M, Fuerst A, Elser F, Berger R, Jauch KW. Outcome of laparoscopic surgery for rectal cancer in 101 patients. Dis Colon Rectum 2003;46:1047-53.

5. Redmond HP, Austin OM, Clery AP, Deasy JM. Safety of doublestapled anastomosis in low anterior resection. Br J Surg 1993;80: 924-7.

6. Bannura GC, Cumsille MA, Barrera AE, Contreras JP, Melo CL, Soto DC. Predictive factors of stenosis after stapled colorectal anastomosis: prospective analysis of 179 consecutive patients. World J Surg 2004;28:921-5.

7. MacRae HM, McLeod RS. Handsewn vs. stapled anastomoses in colon and rectal surgery: a meta-analysis. Dis Colon Rectum 1998; 41:180-9.

8. Koo EJ, Choi HJ, Woo JH, Park KJ, Roh YH, Kim KH, et al. Anastomosis by use of compression anastomosis ring $\left(\mathrm{CAR}^{\mathrm{TN}} 27\right)$ in laparoscopic surgery for left-sided colonic tumor. Int J Colorectal Dis 2012;27:391-6.

9. Tulchinsky H, Kashtan H, Rabau M, Wasserberg N. Evaluation of the NiTi Shape Memory BioDynamix ColonRing ${ }^{\text {m }}$ in colorectal anastomosis: first in human multi-center study. Int J Colorectal Dis 2010;25:1453-8.

10. Buchberg BS, Masoomi H, Bergman H, Mills SD, Stamos MJ. The use of a compression device as an alternative to hand-sewn and stapled colorectal anastomoses: is three a crowd? J Gastrointest Surg 2011;15:304-10.

11. Aggarwal R, Darzi A. Compression anastomoses revisited. J Am Coll Surg 2005;201:965-71.

12. Wullstein C, Gross E. Compression anastomosis (AKA-2) in 
colorectal surgery: results in 442 consecutive patients. Br J Surg 2000;87:1071-5.

13. Thiede A, Geiger D, Dietz UA, Debus ES, Engemann R, Lexer GC, et al. Overview on compression anastomoses: biofragmentable anastomosis ring multicenter prospective trial of 1666 anastomoses. World J Surg 1998;22:78-86.

14. Chen TC, Ding KC, Yang MJ, Chang CP. New device for biofragmentable anastomotic ring in low anterior resection. Dis Colon Rectum 1994;37:834-6.

15. Liang JW, Wang Z, Zhang XM, Zhan DW, Zhou ZX. Animal experimental study of compression anastomosis ring for low anterior resection. Zhonghua Wei Chang Wai Ke Za Zhi 2011;14:333-5.

16. Chun J, Lee D, Stewart D, Talcott M, Fleshman J. Comparison of the compression anastomosis ring (EndoCAR) with a circular stapled anastomosis in a porcine model. Surg Innov 2011;18:23540.

17. Kopelman D, Lelcuk S, Sayfan J, Matter I, Willenz EP, Zaidenstein $\mathrm{L}$, et al. End-to-end compression anastomosis of the rectum: a pig model. World J Surg 2007;31:532-7.

18. Szold A. New concepts for a compression anastomosis: superelastic clips and rings. Minim Invasive Ther Allied Technol 2008;17: 168-71.

19. Dauser B, Herbst F. NITI endoluminal compression anastomosis ring (NITI CAR 27): a breakthrough in compression anastomoses? Eur Surg 2009;41:116-9.

20. Dauser B, Winkler T, Loncsar G, Herbst F. Compression anastomosis revisited: prospective audit of short- and medium-term outcomes in 62 rectal anastomoses. World J Surg 2011;35:192532.

21. Akiyoshi T, Ueno M, Fukunaga Y, Nagayama S, Fujimoto Y, Konishi $\mathrm{T}$, et al. Incidence of and risk factors for anastomotic leakage after laparoscopic anterior resection with intracorporeal rectal transection and double-stapling technique anastomosis for rectal cancer. Am J Surg 2011;202:259-64.

22. Kim JS, Cho SY, Min BS, Kim NK. Risk factors for anastomotic leakage after laparoscopic intracorporeal colorectal anastomosis with a double stapling technique. J Am Coll Surg 2009;209:694701.

23. Ambrosetti P, Francis K, De Peyer R, Frossard JL. Colorectal anastomotic stenosis after elective laparoscopic sigmoidectomy for diverticular disease: a prospective evaluation of 68 patients. Dis Colon Rectum 2008;51:1345-9.

24. Ito M, Sugito M, Kobayashi A, Nishizawa Y, Tsunoda Y, Saito N. Relationship between multiple numbers of stapler firings during rectal division and anastomotic leakage after laparoscopic rectal resection. Int J Colorectal Dis 2008;23:703-7. 